\title{
Optimising the introduction of connected and autonomous vehicles in a public transport system using macro-level mobility simulations and evolutionary algorithms.
}

\author{
HAN, K., CHRISTIE, L.A., ZĂVOIANU, A.-C. and MCCALL, J.
}

(C) 2021 Copyright held by the owner/author(s). This is the author's version of the work. It is posted here for your personal use. Not for redistribution. The definitive Version of Record was published in GECCO '21: proceedings of 2021 Genetic and evolutionary computation conference companion, http://dx.doi.org/10.1145/3449726.3459476. 


\section{Optimising the Introduction of Connected and Autonomous Vehicles in a Public Transport System using Macro-Level Mobility Simulations and Evolutionary Algorithms}

\author{
Kate Han \\ School of Computing, Robert Gordon University \\ Aberdeen, Scotland, UK \\ k.han@rgu.ac.uk \\ Alexandru-Ciprian Zăvoianu \\ School of Computing, Robert Gordon University \\ Aberdeen, Scotland, UK \\ c.zavoianu@rgu.ac.uk
}

\begin{abstract}
The past five years have seen a rapid development of plans and test pilots aimed at introducing connected and autonomous vehicles (CAVs) in public transport systems around the world. Using a real-world scenario from the Leeds Metropolitan Area as a case study, we demonstrate an effective way to combine macro-level mobility simulations based on open data (i.e., geographic information system information and transit timetables) with evolutionary optimisation techniques to discover realistic optimised integration routes for CAVs. The macro-level mobility simulations are used to assess the quality (i.e., fitness) of a potential CAV route by quantifying geographic accessibility improvements using an extended version of Dijkstra's algorithm on an abstract multi-modal transport network.
\end{abstract}

\section{CCS CONCEPTS}

- Theory of computation $\rightarrow$ Random search heuristics; • Applied computing $\rightarrow$ Transportation; • Computing methodologies $\rightarrow$ Discrete space search.

\section{KEYWORDS}

public transport, multi-modal transport, macro-level mobility simulations, reachability isochrones, evolutionary algorithms

\section{ACM Reference Format:}

Kate Han, Lee A. Christie, Alexandru-Ciprian Zăvoianu, and John McCall. 2021. Optimising the Introduction of Connected and Autonomous Vehicles in a Public Transport System using Macro-Level Mobility Simulations and Evolutionary Algorithms. In 2021 Genetic and Evolutionary Computation Conference Companion (GECCO '21 Companion), fuly 10-14, 2021, Lille, France. ACM, New York, NY, USA, 2 pages.

Permission to make digital or hard copies of part or all of this work for personal or classroom use is granted without fee provided that copies are not made or distributed for profit or commercial advantage and that copies bear this notice and the full citation on the first page. Copyrights for third-party components of this work must be honored For all other uses, contact the owner/author(s).

GECCO '21 Companion, Fuly 10-14, 2021, Lille, France

(c) 2021 Copyright held by the owner/author(s).

\author{
Lee A. Christie \\ School of Computing, Robert Gordon University \\ Aberdeen, Scotland, UK \\ 1.a.christie@rgu.ac.uk \\ John McCall \\ School of Computing, Robert Gordon University \\ Aberdeen, Scotland, UK \\ j.mccall@rgu.ac.uk
}

\section{INTRODUCTION AND BACKGROUND}

Recent technological developments regarding connected and autonomous vehicles (CAVs) offer transport authorities novel avenues for the improvement of existing public transport systems (PT) systems [6][5]. For example, impactful gains can be obtained via a niche $\mathrm{CAV}$ deployment on routes where $\mathrm{PT}$ provisioning can bring important social benefits to local communities [2], even if expected passenger volumes are too low to be economically viable.

More specifically, our real-life application scenario is to support the West Yorkshire Combined Authority (WYCA) with automatic optimised route discovery for a CAV-centred pilot project in the district of Adel and Wharfedale - Leeds Metropolitan Area, UK. This route must include Horseforth Train Station (a key PT multimodal hub). The quality of the route is assessed by the improvement of average commuting time across the district when aiming to reach Leeds (Central) Station by 10:00 AM on a workday. The baseline to improve upon is 56 minutes and 19 seconds.

\section{PROPOSED APPROACH}

While employing classic spatial-temporal modelling [4] to construct an abstract transport network from Open Street Map (OSM) and General Transit Feed Specification (GTFS) data, our bespoke macro-level mobility simulation procedure for multi-modal PT systems achieves efficiency gains by:

- allowing for the independent modelling of the static (i.e., roads, pathways, existing PT routes) and transient (i.e., evaluated CAV route) parts of the PT network;

- opting for a reversible graph structure that facilitates the computation of both inbound and outbound accessibility (i.e., $t_{g}$ - the commuting times to/from a fixed destination) using Dijkstra's shortest path algorithm [3] on a set of equallyspaced grid points $g \in G$.

We use a bitstring encoding to represent a given CAV route (i.e., a solution candidate). Each bitstring $x$ is of length 120 - the total number of available PT stops in the district. After decoding the list of selected PT stops (i.e., $L=\left\{i: x_{i}=1\right\}$ ) from $x$, the transient GTFS information of the CAV service associated with route $x$ must be generated prior to running the macro-level mobility simulation and computing the fitness $f(x)$ using Equation 1 . 


$$
f(x)=\left(\frac{1}{|G|} \sum_{g \in G} t_{g}(x)\right) *\left(1+P \cdot \frac{\max \{n(x)-8,0\}}{100}\right)
$$

Based on feedback from WYCA domain experts, the CAV service is constructed under the following assumptions: bi-directional and circular routes starting from Horsforth Station, daily availability between 06:00 AM and 07:00 PM, minimal service frequency of one bus every 10 minutes across all stops, PT stop waiting time of 30 seconds, average CAV travel speed of $32 \mathrm{~km} / \mathrm{h}$, average walking speed of $5 \mathrm{~km} / \mathrm{h}$. A penalty factor of $P \%$ is used inside Equation 1 to model the WYCA suggestion that any CAV route $x$ should ideally be serviceable using at most $n(x)=8$ vehicles.

The optimisations were carried out using a standard genetic algorithm (GA) and a basic estimation of distribution approach the population-based incremental learning (PBIL) algorithm [1] . The two solvers were chosen as they are well-suited to assessing the viability of nature-inspired optimisation on novel realworld problems given their wide-application record, lightweight implementation, and generally robust performance. For each solver we evaluated 36 parameter combinations using 10 independent runs and we experimented with biasing searches towards shorter routes by sampling the initial populations from the distribution $p_{\text {start }}(x)=\prod_{i=1}^{120} p_{\text {start }}\left(x_{i}\right)$, with $0.1 \leq p_{\text {start }}\left(x_{i}=1\right) \leq 0.5$.

\section{RESULTS AND CONCLUSIONS}

We performed an initial series of test runs with the penalty parameter $P$ from Equation 1 set to $0 \%$ and the average number of CAVs - i.e., $\mu(n(x))$ - required by the end-of-the-run solutions / routes was 19.6 for the GA and 16.9 for PBIL. When increasing the penalty factor to $1 \%$ the average number of CAVs required to service the 360 end-of-the-run solutions shown in Figures 1c and 1d dropped to 11.47 in the case of the GA and 8 in the case of PBIL. For $P=1 \%$, average GA solution quality is 50 minutes and 58 seconds, while the average PBIL solution quality is 50 minutes and 31 seconds (i.e., $\approx 11 \%$ improvement over the baseline).

More importantly, for both evolutionary solvers, the best solutions (illustrated in Figures 1a and $1 \mathrm{~b}$ ) require the usage of only $8 \mathrm{CAVs}$ to deliver improvements of $\approx \mathbf{1 3 \%}$. It is noteworthy that both the best GA route and the best PBIL route have an intuitively reasonable South-West $\leftrightarrow$ North-East orientation that aims to correct the poor North-East average commuting time of the baseline by using the CAV service to link the North-East with Horsforth Station along the shortest OSM path. The general ability of both solvers to identify key (North-East) PT stops that must be serviced by an optimal route is evidenced in Figures $1 \mathrm{e}$ and $1 \mathrm{f}$ by the frequency of stop selection among all 360 end-of-the-run solutions.

\section{ACKNOWLEDGMENTS}

The authors would like to acknowledge the support and constructive feedback provided by West Yorkshire Combined Authority transport policy officers.

This research was supported as part of ART-Forum, an Interreg project supported by the North Sea Programme of the European Regional Development Fund of the European Union.

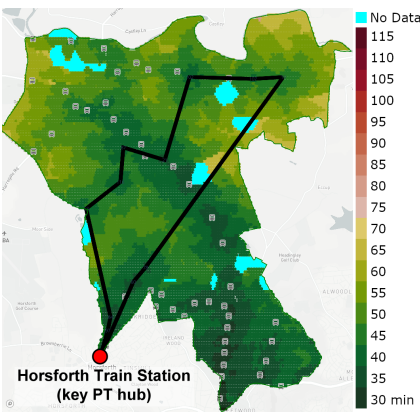

(a) GA best: $f(x)=49 \mathrm{~min}$

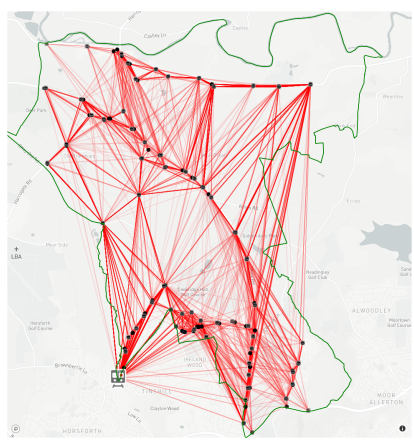

(c) GA: all routes

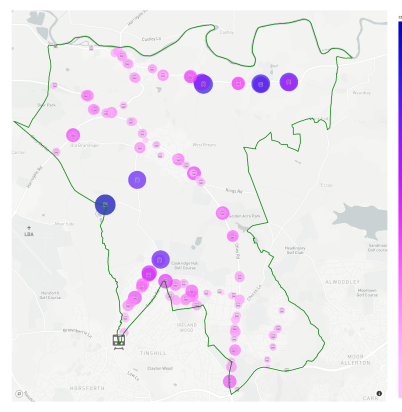

(e) GA: stop frequencies

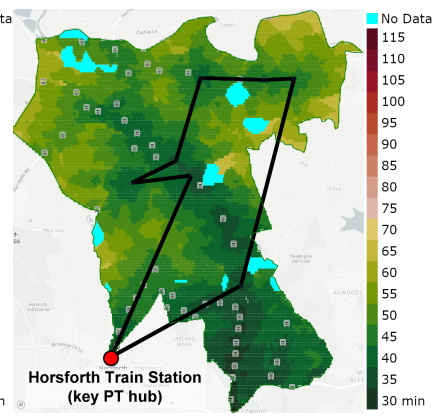

(b) PBIL best: $f(x)=49 \mathrm{~min} 20 \mathrm{~s}$

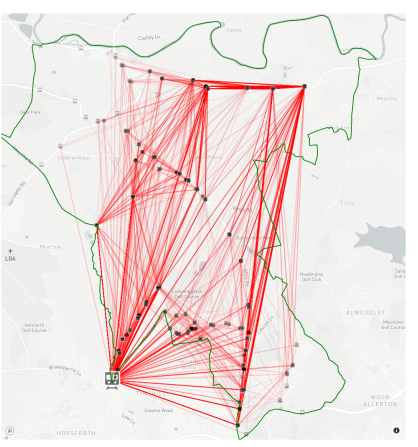

(d) PBIL: all routes

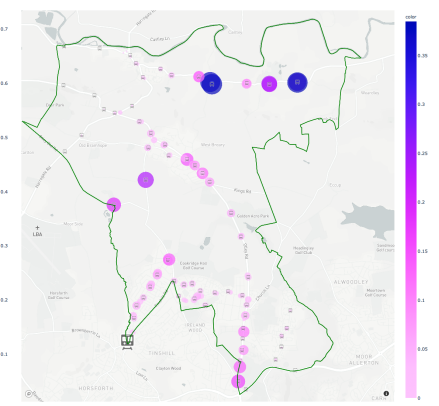

(f) PBIL: stop frequencies
Figure 1: Information regarding all the $360 \mathrm{GA}$ and PBIL endof-the-run solutions when applying a penalty of $P=1 \%$.

\section{REFERENCES}

[1] Shumeet Baluja. 1994. Population-based incremental learning. a method for integrating genetic search based function optimization and competitive learning. Technical Report. Carnegie-Mellon Univ Pittsburgh Pa Dept Of Computer Science.

[2] Søren Risdal Borg and Ditte Bendix Lanng. 2018. Automated busses to mobilize a suburb. In The Royal Geographical Society (with IBG) Annual International Conference, Cardiff University.

[3] Edsger W Dijkstra. 1959. A note on two problems in connexion with graphs. Numerische mathematik 1, 1 (1959), 269-271.

[4] Riccardo Gallotti and Marc Barthelemy. 2015. The multilayer temporal network of public transport in Great Britain. Scientific data 2, 1 (2015), 1-8.

[5] Daniël D Heikoop, JP Nuñez Velasco, Reanne Boersma, Torkel Bjørnskau, and Marjan P Hagenzieker. 2020. Automated bus systems in Europe: a systematic review of passenger experience and road user interaction. Policy Implications of Autonomous Vehicles. Elsevier, The Netherlands (2020), 51-71.

[6] Jonas Meyer, Henrik Becker, Patrick M Bösch, and Kay W Axhausen. 2017. Autonomous vehicles: The next jump in accessibilities? Research in transportation economics 62 (2017), 80-91. 


\section{Optimising the Introduction of Connected and Autonomous Vehicles in a Public Transport System}

using Macro-Level Mobility Simulations and Evolutionary Algorithms

\section{Introduction}

Recent technological developments regarding connected and autonomous vehicles (CAVs) offer transport authorities novel avenues for the improvement of existing public transport systems (PT) systems [1][2]. Impactful gains can be obtained via a niche CAV deployment on routes where PT provisioning can bring important social benefits to local communities [3]. Using a real-world scenario from the Leeds Metropolitan Area as a case study, we demonstrate an effective way to combine macro-level mobility simulations based on open integration routes for CAVs.

\section{Proposed Approach} The macro-level mobility simulations are used to assess the quality (i.e., fitness)
of a potential CAV route by quantifying geographic accessibility improvements on an abstract multi-modal transport network. The computation of both inbound
and outbound accessibility (i.e., $t_{g}-$ the commuting times to/from a fixed destination) is applying an extended version of Dijkstra's shortest path algorithm [4] on a set of equally spaced grid points $g \in G$.

The route optimisations were carried out using a standard genetic algorithm (GA) and the population-based incremental learning (PBIL) algorithm [4] .

We use a bitstring encoding to represent a given CAV route. Each bitstring $x$ length is the total number of PT stops in the district. After decoding the list of selected PT stops, the transient GTFS information of the CAV service associated with route $x$ must be generated prior to running the macro-level
mobility simulation and computing the fitness $f(x)$ using the equation 1 .

$$
f(x)=\left(\frac{1}{|G|} \sum_{g \in G} t_{g}(x)\right) *\left(1+P \frac{\max \{n(x)-8,0\}}{100}\right)(1)
$$

For each solver we evaluated 36 parameter combinations using 10 independent runs and we experimented with biasing searches towards shorter routes by sampling the initial populations from the distribution in equation 2.

$p_{\text {start }}(x)=\prod_{i-1}^{120} p_{\text {start }}\left(x_{i}\right)$, with $0.1 \leq p_{\text {start }}\left(x_{i}=1\right) \leq 0.5(2)$

\section{RTी ROBERT GORDON UNIVERSITY ABERDEEN}

Kate Han, Lee A. Christie, Alexandru-Ciprian Zăvoianu, John McCal school of Computing, Robert Gordon University Aberdeen, Sco
$\{$ \{k.han, la.christie, c.zavoianu, i.mccall\}@rgu.ac.uk

\section{Figures and Results}

We performed an initial series of test runs with the penalty parameter $P$ from Equation 1 set to $0 \%$ and the average number of $\mathrm{CAVs}-$ i.e., $\mu(n(x))$ - required by the end-of-the-run solutions / routes was 19.6 for the GA and 16.9 for PBIL. When increasing the penalty factor to $1 \%$ the average number of CAVs required to service the 360 end-of-the-run solutions shown in Figures $1 \mathrm{c}$ and $1 \mathrm{~d}$ dropped 11.47 in the case of the GA and 8 in the case of PBIL. For $P=1 \%$, average GA solution quality is 50 minutes and 58 seconds, while the average PBIL solution quality is 50 minutes and 31 seconds (i.e., $\approx 11 \%$ improvement over the baseline).
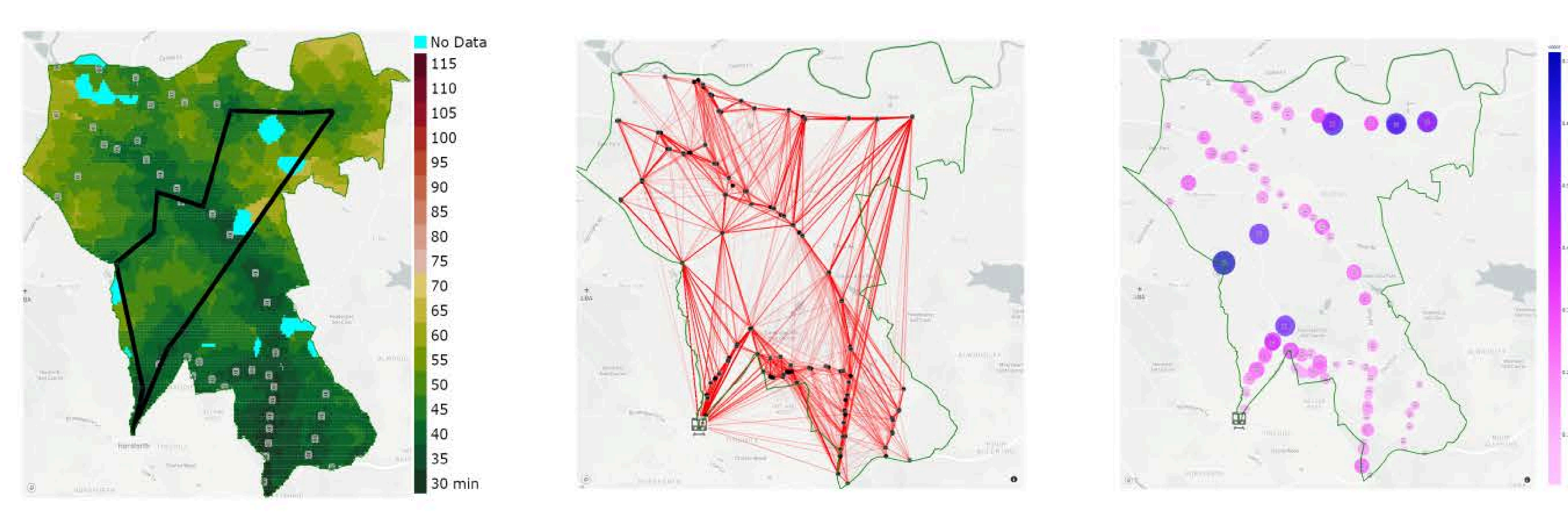

(a) GA best: $f(x)=49 \mathrm{~min}$

(c) GA: all routes

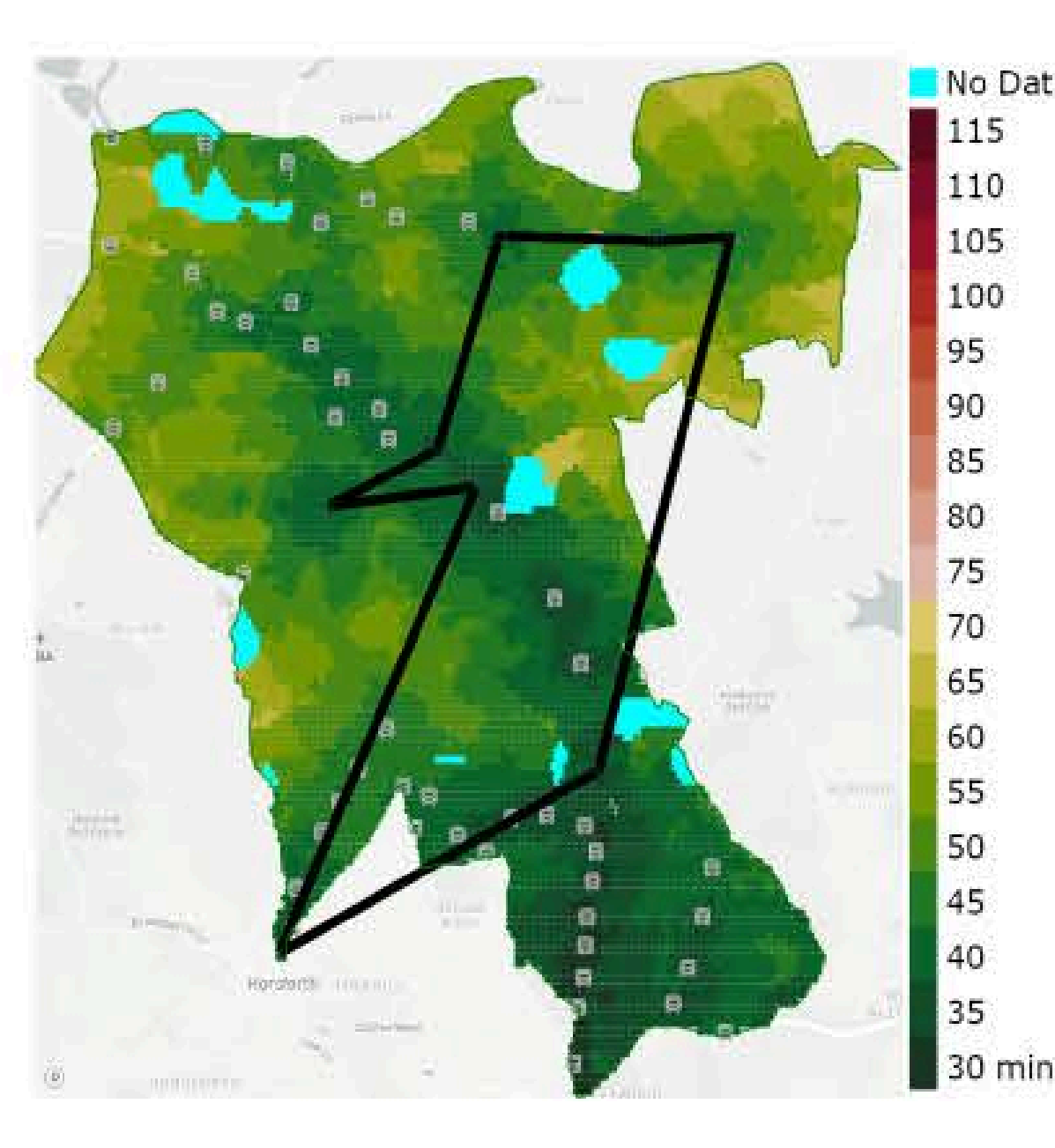

(b) PBIL best: $f(x)=49 \mathrm{~min}$

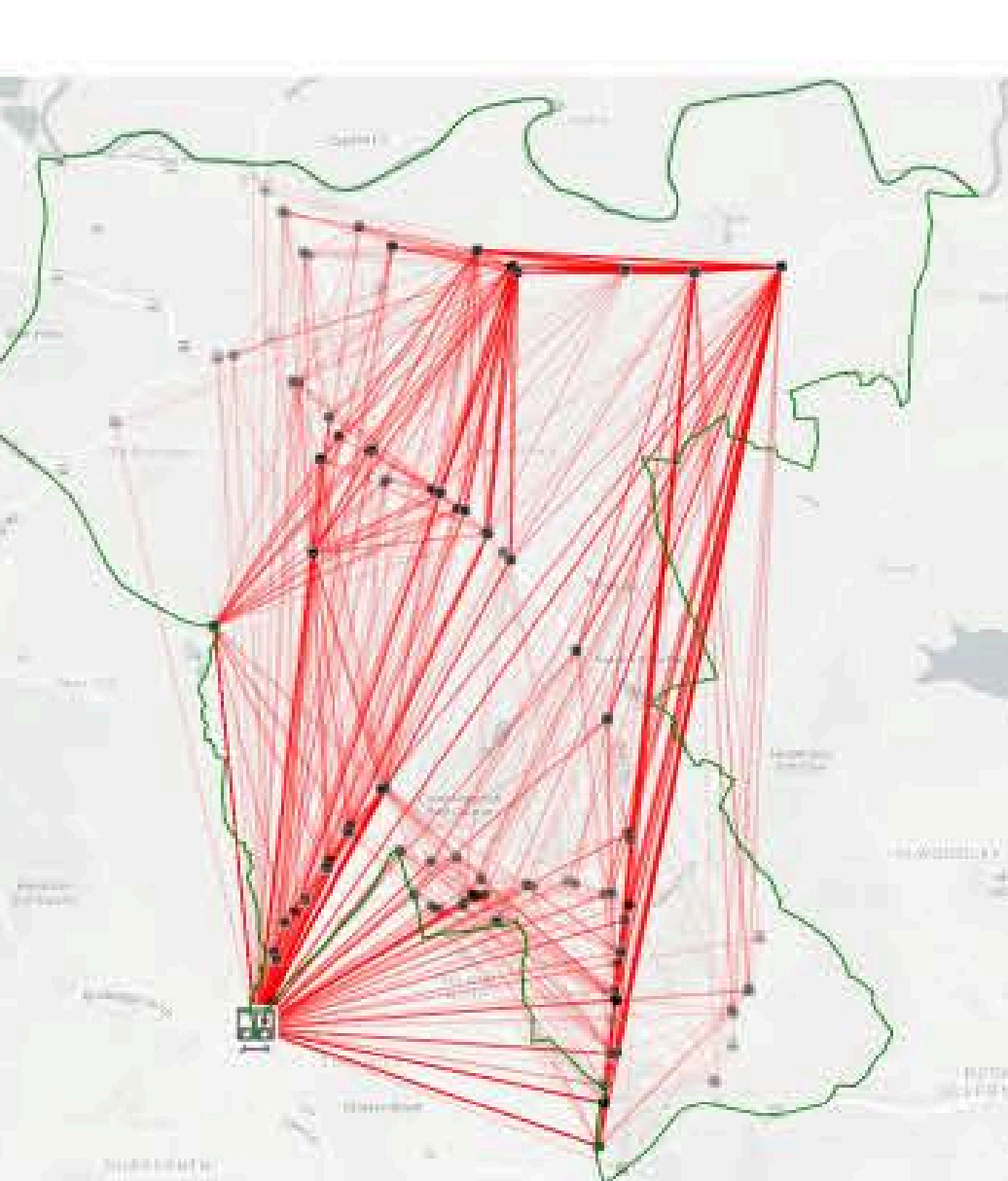

(d) PBLL: all routes (e) GA: stop frequencies

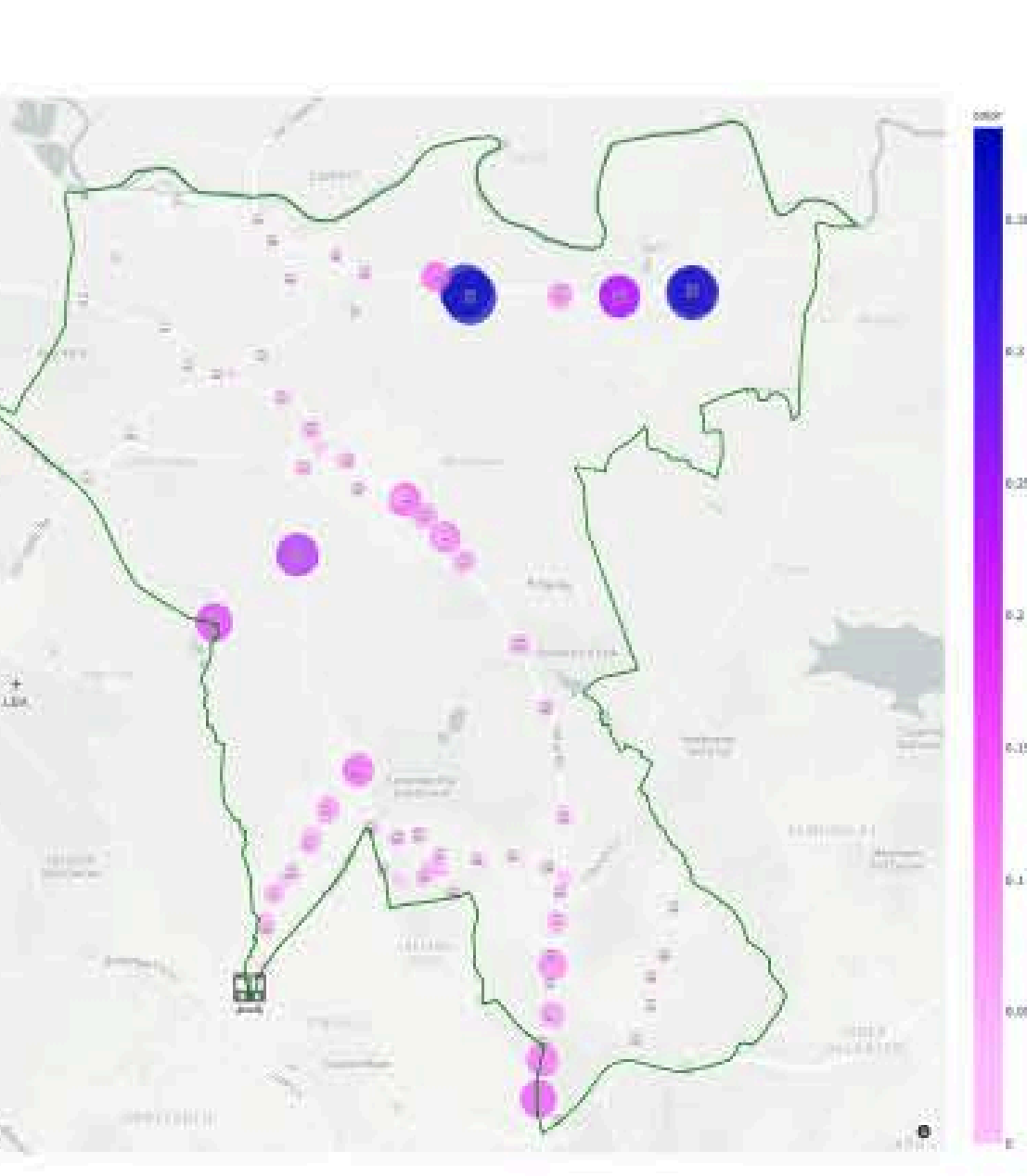

(f) PBIL: stop frequencies
Figure 1: Information regarding all the $360 \mathrm{GA}$ and PBIL end-of-the-run solutions when applying a penalty of $P=1 \%$.

\section{interreg North Sea Region ART-Forum

Conclusion

More importantly, for both evolutionary solvers, the best solutions (illustrated in Figures $1 \mathrm{a}$ and $1 \mathrm{~b}$ ) require the usage of only $8 \mathrm{CAVs}$ to deliver improvements of $\approx 13 \%$. It is noteworthy that both the best GA route and the best PBIL route have an intuitively reasonable South-West $\leftrightarrow$ North-East orientation that aims to correct the poor North-East average commuting time of the baseline by using the CAV service to link the North-East with Horsforth Station along the shortest OSM path. The general ability of both solvers to identify key (North-East) PT $1 \mathrm{f}$ by the frequency of stop selection among all 360 end-of-the-run solutions.

\section{Acknowledgments}

The authors would like to acknowledge the support and constructive feedback provided by West Yorkshire Combined Authority trans- port policy officers. This research was supported as part of ART-Forum, an Interreg project supported by the Nort Development Fund of the European Union.

\section{References}

[1] Jonas Meyer et al. 2017. Autonomous vehicles: The next jump in accessibitities? Research in transportation economics 62 (2017), 80-91.

[2] Daniel D Heikoop et al.. 2020. Automated bus systems in Europe: a systematic review of passenger experience and road user interaction. Policy [3] Saren Risdal Borg and Ditte Bendix Lanng. 2018. Automs (2020), 51-71. [3] Søren Risdal Borg and Ditte Bendix Lanng. 2018. Automated busses to Internation 14] Edsger W Dijkstra. 1959. A note on two

A note on two problems in connexion with graphs. Numerische mathematik 1, 1 (1959), 269-271.

[5] Shumeet Baluja. 1994. Population-based incremental learning. a method for inte grating genetic search based function optimization and competitive learning. Technical Report. Carnegie-Mellon Univ Pittsburgh Pa Dept Of Computer Science.

\section{A.R.T.Forum}

